\title{
IL-12 and IL-23 Production in Toxoplasma gondii- or LPS- Treated Jurkat T Cells via PI3K and MAPK Signaling Pathways
}

\author{
Hassan Ahmed Hassan Ahmed Ismail ${ }^{1, \dagger}$, Byung-Hun Kang ${ }^{2, \dagger}$, Jae-Su Kim ${ }^{1, \dagger}$, Jae-Hyung Lee ${ }^{1,3}$, In-Wook Choi', \\ Guang-Ho Cha', Jae-Min Yuk', Young-Ha Lee ${ }^{1, *}$ \\ ${ }^{1}$ Department of Infection Biology, Chungnam National University School of Medicine, Daejeon 34134, Korea; 2 Department of Obstetrics and \\ Gynecology, Chungnam National University School of Medicine, Daejeon 34134, Korea; ' ${ }^{3}$ epartment of Biomedical Science, Chungnam National \\ University Graduate School, Daejeon 34134, Korea
}

\begin{abstract}
IL-12 and IL-23 are closely related in structure, and have been shown to play crucial roles in regulation of immune responses. However, little is known about the regulation of these cytokines in T cells. Here, we investigated the roles of PI3K and MAPK pathways in IL-12 and IL-23 production in human Jurkat T cells in response to Toxoplasma gondii and LPS. IL-12 and IL-23 production was significantly increased in T cells after stimulation with T. gondii or LPS. T. gondii and LPS increased the phosphorylation of AKT, ERK1/2, p38 MAPK, and JNK1/2 in T cells from 10 min post-stimulation, and peaked at 30-60 min. Inhibition of the PI3K pathway reduced IL-12 and IL-23 production in T. gondii-infected cells, but increased in LPS-stimulated cells. IL-12 and IL-23 production was significantly reduced by ERK1/2 and p38 MAPK inhibitors in T. gondii- and LPS-stimulated cells, but not in cells treated with a JNK1/2 inhibitor. Collectively, IL-12 and IL-23 production was positively regulated by PI3K and JNK1/2 in T. gondii-infected Jurkat cells, but negatively regulated in LPS-stimulated cells. And ERK1/2 and p38 MAPK positively regulated IL-12 and IL-23 production in Jurkat T cells. These data indicate that $T$. gondii and LPS induced IL-12 and IL-23 production in Jurkat T cells through the regulation of the PI3K and MAPK pathways; however, the mechanism underlying the stimulation of IL-12 and IL-23 production by T. gondii in Jurkat T cells is different from that of LPS.
\end{abstract}

Key words: Toxoplasma gondii, LPS, IL-12, IL-23, PI3K/AKT, MAPK pathway

\section{INTRODUCTION}

Toxoplasma gondii is a ubiquitous apicomplexan parasite that infects an estimated one-third of the global population [1]. Although infection of immunocompetent humans is usually asymptomatic, T. gondii may lead to life-threatening disease in fetuses, or in immunocompromised patients after activation of dormant parasites [1]. This parasite induces a type $1 \mathrm{im}$ mune response characterized by the production of interferon (IFN)- $\gamma$ by CD4 $4^{+}$and $\mathrm{CD} 8^{+}$T-cells $[2,3]$. Lipopolysaccharide (LPS) is an essential component of the outer membrane of gram-negative bacteria, and is also known as lipoglycan and endotoxin. LPS is one of the most potent innate-immune-acti-

- Received 11 July 2017, revised 14 November 2017, accepted 24 November 2017.

*Corresponding author (yhalee@cnu.ac.kr)

†These authors contributed equally to this work.

(c) 2017, Korean Society for Parasitology and Tropical Medicine

This is an Open Access article distributed under the terms of the Creative Commons Attribution Non-Commercial License (http://creativecommons.org/licenses/by-nc/4.0) which permits unrestricted non-commercial use, distribution, and reproduction in any

medium, provided the original work is properly cited. vating stimuli, and it induces production of various cytokines, including tumor necrosis factor (TNF)- $\alpha$, IL-1 family, IL-6, and the IL-12 family [4,5]. IL-12 family cytokines are composed of IL-12, IL-23, and IL-27; however, the mechanisms underlying the production and regulation of the IL-12 family cytokines in human T-cells are unclear.

IL-12 and IL-23 have been shown to play crucial roles in the regulation of innate and adaptive immune responses [6]. Macrophages and dendritic cells (DCs) are the major sources of both IL-12 and IL-23, which act on receptors primarily expressed by T cells, NK cells, and NKT cells [7]. IL-12 is a key factor in the induction of $\mathrm{T}$ cell-dependent and independent activation of macrophages and natural killer (NK) cells, and it plays a central role in Th1 development [6]. IL-23, like IL-12, is a pro-inflammatory cytokine produced by activated DCs and macrophages in response to microbial pathogens [8]. Both IL 12 and IL-23 are also critical for antibacterial host defense and are linked to autoimmune diseases, including multiple sclerosis and arthritis $[6,8,9]$. Lieberman et al. [10] indi- 
cated that IL-12, not IL-23, plays a dominant role in resistance to toxoplasmosis but, in the absence of IL-12, IL-23 can provide a limited mechanism of resistance to this infection. There were some reports about IL-12 and IL-23 production in macrophages and DCs after T. gondii infection [10-12]; however, few studies have addressed the effect of T. gondii or LPS on these cytokine productions by $\mathrm{T}$ cells.

Phosphatidylinositol 3-kinase (PI3K) is a family of enzymes involved in cellular functions such as growth, proliferation, differentiation, motility, survival, and intracellular trafficking. This pathway is activated by a combination of ligands such as LPS, and various cell surface receptors such as Toll like receptors (TLRs), insulin receptor, estrogen receptor, and numerous cytokine receptors [13]. Mitogen-activated protein kinase (MAPK) signaling pathways are also involved in diverse cellular responses in all eukaryotic organisms including inflammation, proliferation, differentiation, and apoptosis. The MAPK family includes extracellular signal-regulated kinase $1 / 2$ (ERK1/2), p38 MAPK, and c-Jun N-terminal kinase 1/2 (JNK1/2) [13]. T. gondii activates PI3K, leading to phosphorylation of the downstream serine/threonine kinase AKT (also known as protein kinase B) $[10,12,14]$. Also, the MAPK signaling pathway is involved in the production of IL-12 or IL-23 in response to $T$. gondii $[5,10,14,15]$. The signaling pathways associated with IL-12 and IL-23 production in T. gondii infected macrophages and DCs have been reported; however, the mechanisms underlying IL-12 and IL-23 production in T cells after T. gondii or LPS stimulation are unclear.

IL-12 and IL-23 are involved in the regulation of both innate and adaptive immunity; however, there is limited information on the signaling sequences related to their production in T cells. Therefore, to identify the roles of the PI3K and MAPK pathways in IL-12 and IL-23 production in T cells, Jurkat $\mathrm{T}$ cells were stimulated with live T. gondii and LPS, and the production of IL-12 and IL-23 was evaluated using specific inhibitors of the PI3K and MAPK pathways.

\section{MATERIALS AND METHODS}

\section{Reagents and antibodies}

Antibodies against phosphorylated ERK1/2 (p-ERK1/2), total ERK1/2 (ERK1/2), phosphorylated p38 MAPK (p-p38 MAPK), total p38 MAPK (p38 MAPK), phosphorylated JNK1/2 (p-JNK1/2), total JNK (JNK1/2), phosphorylated AKT (p-AKT), total AKT (AKT), and $\alpha$-tubulin were purchased from
Cell Signaling Technology Inc. (Danvers, Massachusetts, USA). The ERK1/2 inhibitor PD98059, p38 MAPK inhibitor SB203580, and JNK inhibitor SP600125 were obtained from Calbiochem (San Diego, California, USA). The LPS and PI3K inhibitors, LY294002 and wortmannin, were obtained from Sigma-Aldrich (St. Louis, Missouri, USA). Secondary antibodies, antirabbit-horseradish peroxidase (HRP) and anti-mouse-HRP, were purchased from Sigma-Aldrich.

\section{Cell line and parasites}

A human lymphoblast-like cell line, Jurkat T cells (American Type Culture Collection, Rockville, Maryland, USA), was cultured in RPMI 1640 medium (Gibco-Invitrogen, Carlsbad, California, USA) supplemented with $10 \%$ heat-inactivated fetal bovine serum (FBS; Gibco BRL), $2 \mathrm{mM}$ glutamine, $25 \mathrm{mM}$ (4-[2-hydroxyethyl]-1-piperazineethanesulfonic acid [HEPES; Gibco-Invitrogen]), and antibiotic-antimycotic. The cells were incubated at $37^{\circ} \mathrm{C}$ in a $5 \% \mathrm{CO}_{2}$, humidified atmosphere.

Tachyzoites of $T$. gondii RH were maintained on human retinal pigment epithelium cells (ARPE 19 cells) by biweekly passage in Dulbecco's modified Eagle's medium (DMEM; Welgene, Daegu, Korea) supplemented with 10\% heat-inactivated FBS, 2 mM glutamine, $25 \mathrm{mM}$ HEPES, and antibiotic-antimycotic. To isolate T. gondii tachyzoites, a suspension of T. gondiicontaining scrapped cells was passed through a 27-gauge needle and centrifuged at $100 \mathrm{~g}$ for $5 \mathrm{~min}$ to pellet the host cells. The supernatant was centrifuged at 1,350 g for $10 \mathrm{~min}$ in Percoll (Sigma-Aldrich) to pellet the tachyzoites. Cell viability was deter $\neg$ mined by trypan blue dye-exclusion assay.

\section{Stimulation of Jurkat T cells with T. gondii tachyzoites or LPS}

Human Jurkat $\mathrm{T}$ cells were seeded on 24-well plates (for enzyme-linked immunosorbent assay [ELISA]) or $100 \mathrm{~mm}$ culture dishes (for western blotting) at various densities, and grown to confluence at $37^{\circ} \mathrm{C}$ in $5 \% \mathrm{CO}_{2}$. Jurkat $\mathrm{T}$ cells were stimulated by adding fresh live T. gondii RH tachyzoites (multiplicity of infection [MOI] of 1) or $0.2 \mu \mathrm{g} / \mathrm{mL}$ LPS. IL-12 and IL-23 production and PI3K/AKT and MAPK subtype phosphorylation in T. gondii- and LPS-stimulated Jurkat T cells were evaluated by ELISA and western blotting. To investigate the roles of the PI3K/AKT and MAPK signaling pathways in the production of IL-12 and IL-23, T. gondii - and LPS-stimulated cells were pretreated with PI3K or MAPK inhibitors and cytokine levels in the culture supernatants were determined. 


\section{ELISA for IL-12 and IL-23}

To determine IL-12 and IL-23 levels in culture supernatants, Jurkat T cells were stimulated with $T$. gondii or LPS with or without specific inhibitors. Culture supernatants were harvested at the indicated time points and stored at $-70^{\circ} \mathrm{C}$ until analysis. Human IL-12 p70 and IL-23 were detected using an ELISA kit according to the manufacturer's instructions (R\&D Systems, Inc., Minneapolis, Minnesota, USA). Cytokine concentrations in the culture supernatants were calculated from standard curves generated using recombinant cytokines, and were expressed in picograms per milliliter.

\section{Western blot analysis}

Jurkat T cells were stimulated with T. gondii or LPS with or without pretreatment with specific inhibitors. At the indicated time points, cells were washed with phosphate-buffered saline (PBS), and proteins were extracted using PRO-PREP ${ }^{\mathrm{TM}}$ Protein Extraction Solution (iNtRON Biotechnology, Seoul, Korea) for $15 \mathrm{~min}$ on ice. After centrifugation at $4^{\circ} \mathrm{C}$ for $15 \mathrm{~min}$ at 14,000 $g$, the supernatants were collected, and equal amounts of protein from each sample were resolved by standard SDS-PAGE and transferred to polyvinylidene difluoride membranes. The membranes were blocked with 5\% skim milk in Tris-buffered saline (20 mM Tris, $137 \mathrm{mM} \mathrm{NaCl}$, pH 7.6) containing 0.1\% Tween 20 (TBST). After washing once in TBST, the membranes were incubated overnight at $4^{\circ} \mathrm{C}$ with anti-p-AKT, anti-AKT, anti-p-ERK1/2, anti-ERK1/2, anti-p38 MAPK, anti-p-p38 MAPK, anti-p-JNK1/2, anti-JNK1/2, and $\alpha$-tubulin antibodies (all from Cell Signaling Technology) diluted in 5\% bovine serum albumin in TBST. Following 3 washes in TBST, the membranes were incubated for $120 \mathrm{~min}$ with HRP-conjugated anti-mouse or anti-rabbit IgG (Sigma-Aldrich) diluted at 1:10,000 in incubation buffer. After extensive washing, the bound antibodies were visualized by enhanced chemiluminescence (Amersham Biosciences, Freiburg, Germany).

\section{Statistical analysis}

Results are expressed as means \pm SDs of at least 3 independent experiments unless otherwise indicated. The statistical significance of the data was determined by unpaired Student's $t$-test. $P$-values of less than 0.05 were deemed to indicate statistical significance.

\section{RESULTS}

\section{T. gondii and LPS induced IL-12 and IL-23 production in Jurkat $T$ cells}

To assess the effect of T. gondii or LPS on IL-12 and IL-23 production, Jurkat $\mathrm{T}$ cells were stimulated with live $\mathrm{T}$. gondii or LPS for $24 \mathrm{hr}$. IL-12 and IL-23 production in T. gondii-stimulated cells increased over time (Fig. 1) and significantly increased from $2 \mathrm{hr}$ after stimulation $(P<0.05)$. At 12 and $24 \mathrm{hr}$ poststimulation, the levels of IL-12 and IL-23 production in T. gondii-infected T cells had increased in a dose-dependent manner. In LPS-stimulated T cells, the patterns of IL-12 and IL-23 production were similar with $T$. gondii-stimulated cells. However,
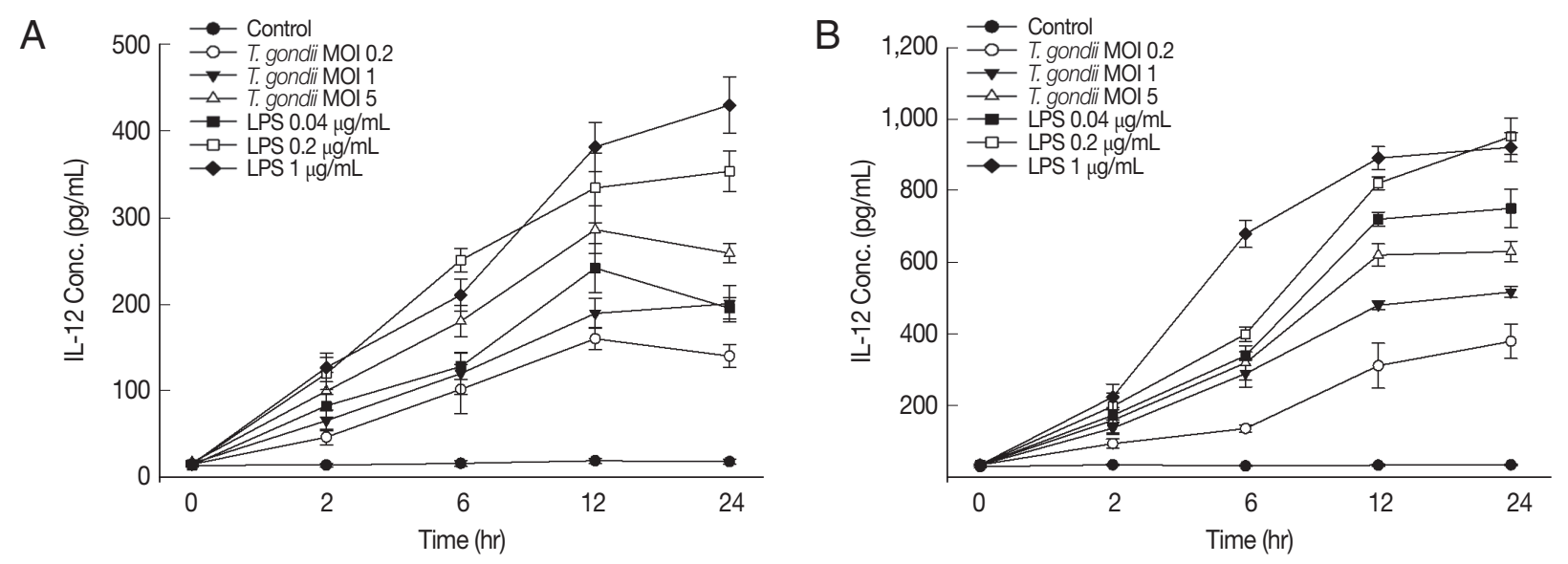

Fig. 1. T. gondii and lipopolysaccharide (LPS) induce IL-12 and IL-23 production in human Jurkat T cells in a dose dependent manner. Human Jurkat T cells were stimulated with live T. gondii (multiplicity of infection of 1$)$ or LPS $(0.2 \mu \mathrm{g} / \mathrm{ml})$. Culture supernatants were collected at the indicated time points. IL-12 (A) and IL-23 (B) levels in the culture supernatants were determined by ELISA. One representative result of the 2 independent replicates is shown. 
A

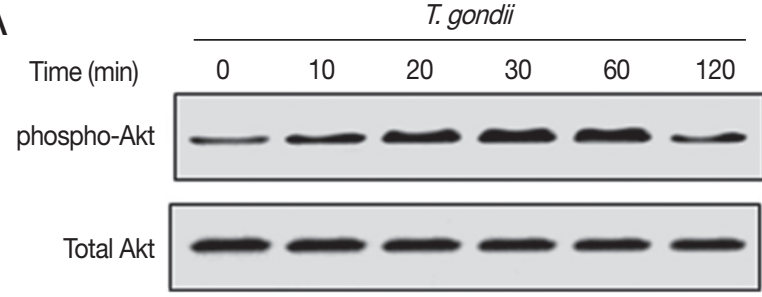

B

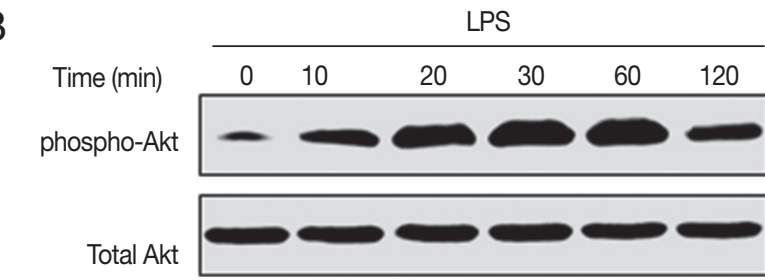

C

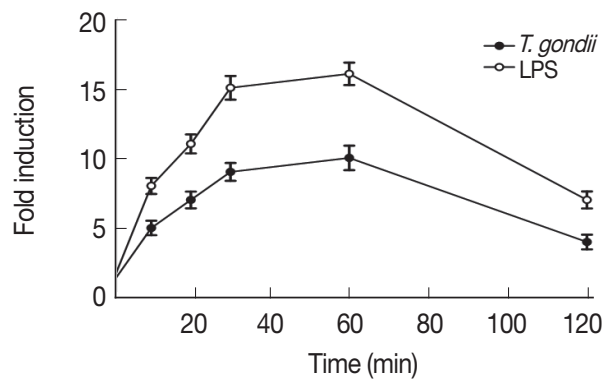

Fig. 2. T. gondii and LPS rapidly activate the phosphatidylinositol 3-kinase (PI3K)/AKT signaling pathway. (A, B) Cell lysates were prepared at the indicated times after T. gondii infection (A) or LPS stimulation (B), and subjected to western blotting for anti-phospho-AKT. The blots were stripped and re-probed with antibodies to total AKT. (C) Densitometric analysis. One representative result of the 2 independent replicates is shown.

their concentrations were significantly higher in LPS-stimulated cells than in T. gondii-infected cells at each time point $(P<0.05)$. Therefore, T. gondii induced IL-12 and IL-23 production in Jurkat $\mathrm{T}$ cells in a time- and dose-dependent manner, similar to LPS-stimulated Jurkat $\mathrm{T}$ cells.

\section{T. gondii and LPS activate the PI3K/AKT signaling pathway in Jurkat $T$ cells}

We next determined whether T. gondii and LPS activate the PI3K/AKT pathway in Jurkat $\mathrm{T}$ cells. Jurkat $\mathrm{T}$ cells exhibited transient activation of AKT in response to T. gondii or LPS (Fig. 2). AKT was phosphorylated from 10 min after infection, reached a plateau at $1 \mathrm{hr}$, and decreased thereafter (Fig. 2A, C). Similar results were observed in LPS-stimulated cells (Fig. 2B, C). Therefore, stimulation of Jurkat T cells with T. gondii or LPS results in activation of the PI3K/AKT pathway.
IL-12 and IL-23 production was positively regulated by $\mathrm{PI} 3 \mathrm{~K}$ in T. gondii-infected Jurkat T cells, but negatively regulated in LPS-treated ones.

To investigate the role of the PI3K/AKT signaling pathway in T. gondii- and LPS-induced IL-12 and IL-23 production, Jurkat $\mathrm{T}$ cells were pretreated for $1 \mathrm{hr}$ with the PI3K/AKT inhibitors, LY294002 or wortmannin, followed by T. gondii or LPS stimulation. PI3K inhibitors decreased IL-12 and IL-23 production in T. gondii-infected cells in a dose-dependent manner (Fig. 3A, C, E, G). Pretreatment with 1 or $10 \mu \mathrm{M}$ LY294002 or wortmannin significantly inhibited the production of IL-12 and IL-23 in T. gondii infected cells $(P<0.05)$. In contrast, LPS-induced IL-12 and IL-23 production was dose dependently increased by pretreatment with either of the PI3K inhibitors (Fig. 3B, D, F, H). Pretreatment with $10 \mu \mathrm{M}$ LY294002 or wortmannin significantly increased the production of IL-12 and IL-23 in comparison to LPS-stimulated cells $(P<0.05)$. These data indicate that the PI3K/AKT pathway upregulates IL-12 and IL 23 production by Jurkat T cells stimulated with T. gondii, whereas IL12 and IL-23 production is downregulated by PI3K/AKT pathway in LPS-stimulated Jurkat T cells.

\section{T. gondii and LPS induce ERK1/2, p38 MAPK, and JNK1/2 phosphorylation in Jurkat T cells}

We analyzed the activation of MAPK subtypes by T. gondii and LPS. Phosphorylation of ERK1/2, p38 MAPK, and JNK1/2 was significantly increased from 10-20 min following $T$. gondii or LPS stimulation, and peaked at 30-60 min, thereafter decreased to background levels at 120 min (Fig. 4). The phosphorylation patterns of ERK1/2, p38 MAPK, and JNK1/2 in T. gondii-infected cells were similar to those of LPS-stimulated cells. ERK1/2 and p38 MAPK phosphorylation was occurred more rapidly and reached a higher level in LPS-stimulated Jurkat $\mathrm{T}$ cells than those of $T$. gondii-stimulated ones; however, $T$. gondii and LPS stimulation resulted in similar levels of JNK1/2 phosphorylation. Therefore, stimulation of Jurkat $\mathrm{T}$ cells with T. gondii or LPS results in rapid activation of ERK1/2, p38 MAPK, and JNK1/2.

The roles of MAPK subtypes in the regulation of IL-12 and IL-23 production in Jurkat T cells

To assess the involvement of the MAPK subtype pathways in the regulation of IL-12 and IL-23 production, we used inhibitors of each signaling pathway. IL-12 and IL-23 production in T. gondii- or LPS-stimulated Jurkat $\mathrm{T}$ cells was significantly de- 
A

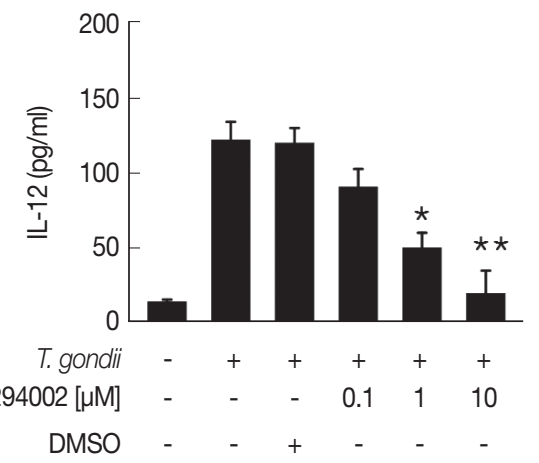

C

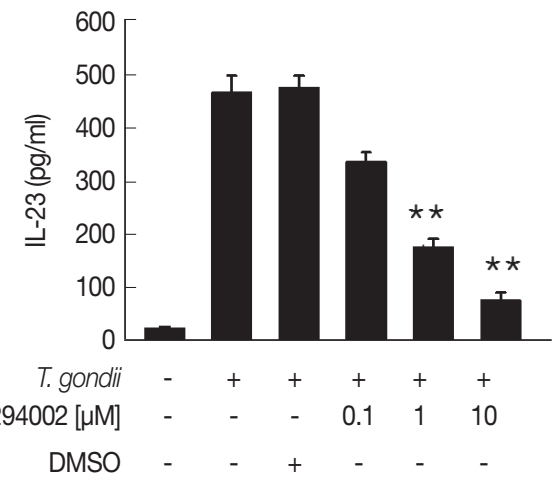

E

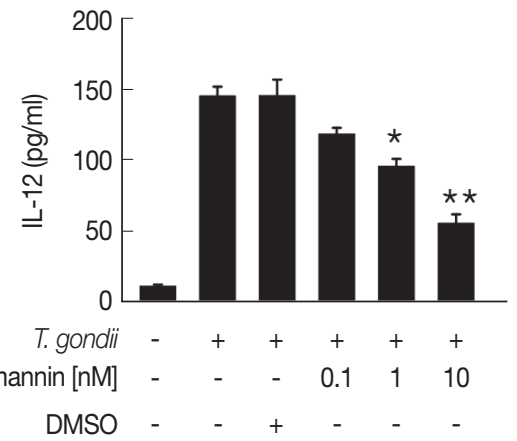

G

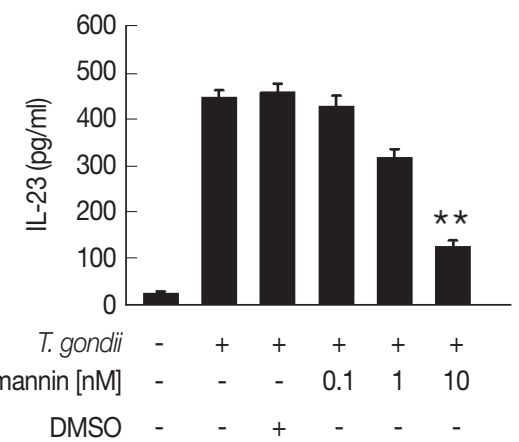

B

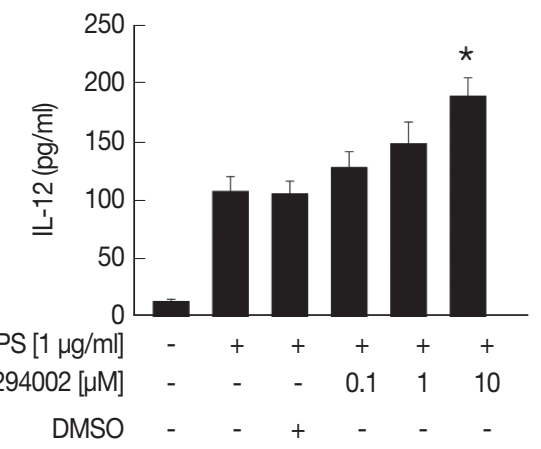

D

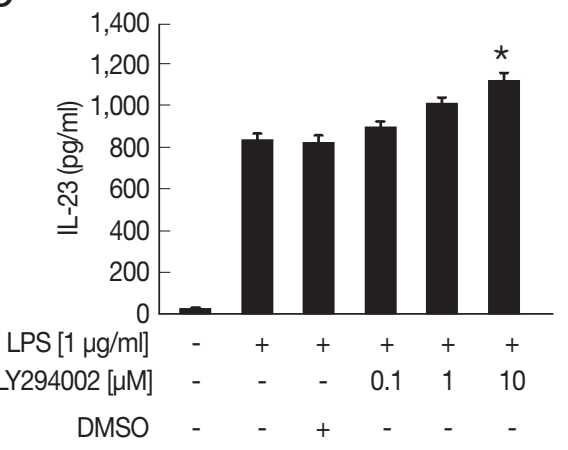

F

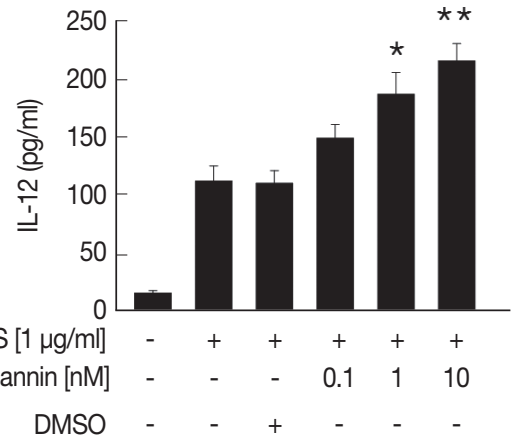

$\mathrm{H}$

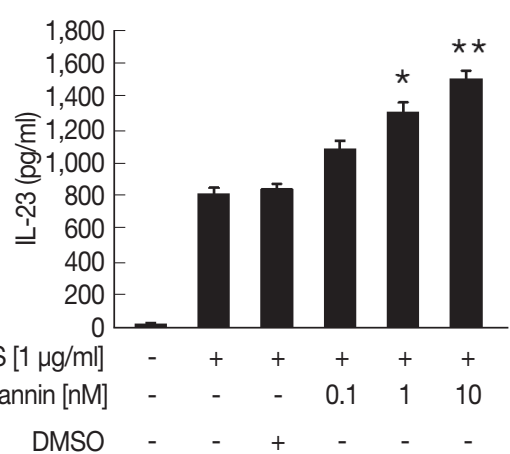

Fig. 3. T. gondii and LPS differentially regulate IL-12 and IL-23 production in human Jurkat T cells. Cells were pre-incubated with the PI3K inhibitors LY294002 and wortmannin for $1 \mathrm{hr}$, and then infected with T. gondii (A, C, E, and G) or stimulated with LPS (B, D, F, and H) for $12 \mathrm{hr}$. IL-12 and IL-23 levels in the culture supernatants were analyzed in duplicate by ELISA. One representative result of the 2 independent replicates is shown. Denotes $P<0.05^{\star}$ and $P<0.01^{\star \star}$, compared to media-treated control.

creased by the ERK1/2 and p38 MAPK inhibitors in a dose-dependent manner; especially, treatment with $10 \mu \mathrm{M}$ of $\mathrm{p} 38$
MAPK inhibitor almost abolished IL-12 and IL 23 production in T. gondii-infected T cells (Fig. 5A-H). Pretreatment with the 
A
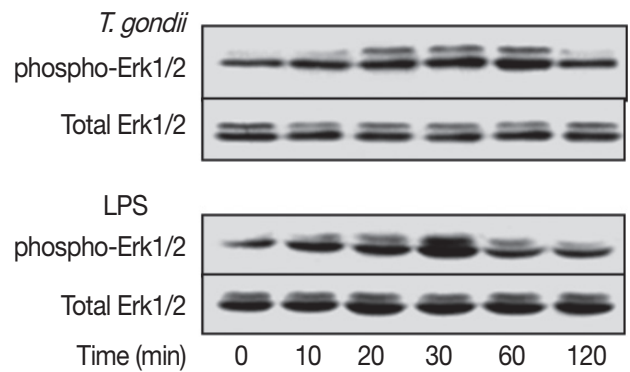

C
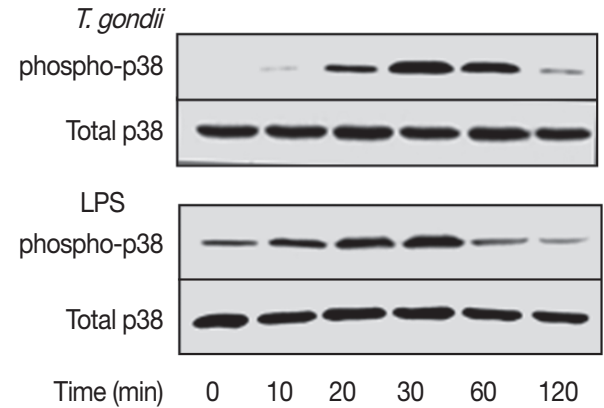

$\mathrm{E}$
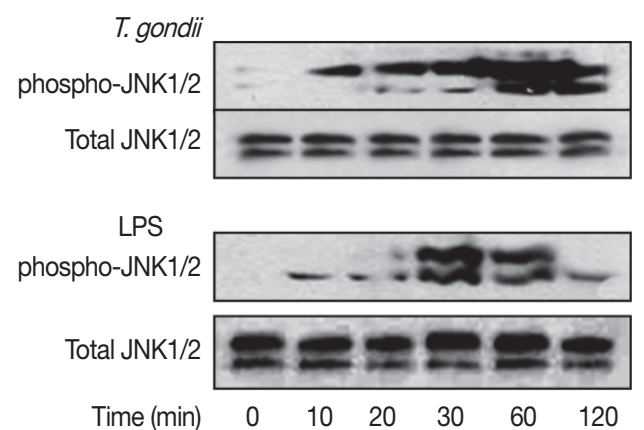

B

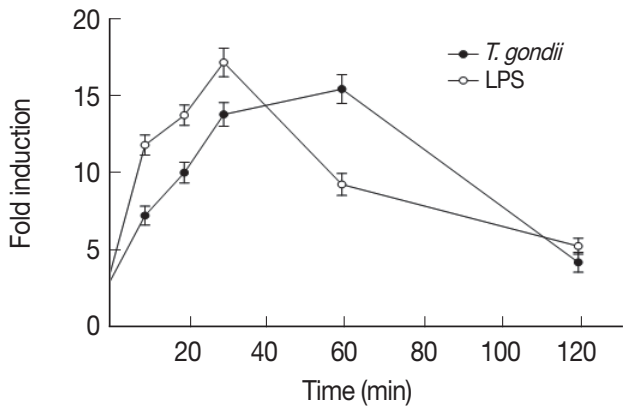

D

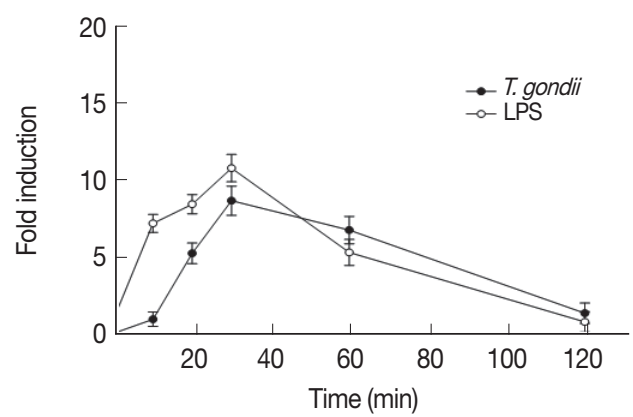

$\mathrm{F}$

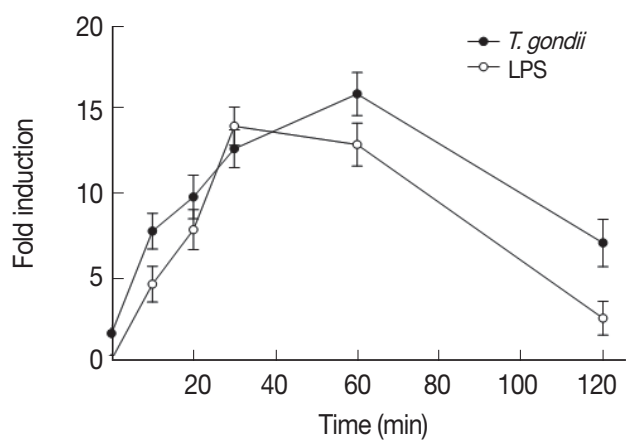

Fig. 4. T. gondii and LPS induce transient phosphorylation of ERK1/2, p38 MAPK, and JNK1/2 in human Jurkat T cells. Human Jurkat $\mathrm{T}$ cells were stimulated with $\mathrm{T}$. gondii RH or LPS at the indicated time points. Western blots were incubated overnight with monoclonal antibodies against phospho ERK1/2, total ERK1/2 (A), phospho-p38 MAPK, total p38 MAPK (C), phospho-JNK1/2, and total JNK1/2 (E). Densitometric analysis of the levels of p-ERK1/2 (B), p-p38 MAPK (D), and p-JNK1/2 (F). One representative result of the 2 independent replicates is shown.

JNK1/2 inhibitor PD98059 decreased IL-12 and IL-23 production in T. gondii-infected cells significantly, whereas LPS stimulation increased IL 12 and IL-23 production (Fig. 5I-L). These results indicate that IL-12 and IL-23 production is positively regulated by the ERK1/2 and 338 MAPK pathways in response to T. gondii or LPS. However, the roles of JNK1/2 pathway on IL-12 and IL-23 production in Jurkat T cells differed depending on the stimulus applied.

\section{DISCUSSION}

In this study, we investigated the roles of the PI3K/AKT and MAPK signaling pathways in IL-12 and IL-23 production by human Jurkat T cells in response to T. gondii and LPS. IL-12 and IL23 production was significantly increased in a time- and dosedependent manner in Jurkat $\mathrm{T}$ cells after stimulation with $T$. gondii or LPS. Jurkat T cells exposed to T. gondii and LPS exhibited rapid phosphorylation of AKT, ERK1/2, p38 MAPK, and JNK1/2. Interestingly, IL-12 and IL-23 production by T. gondii- 
A

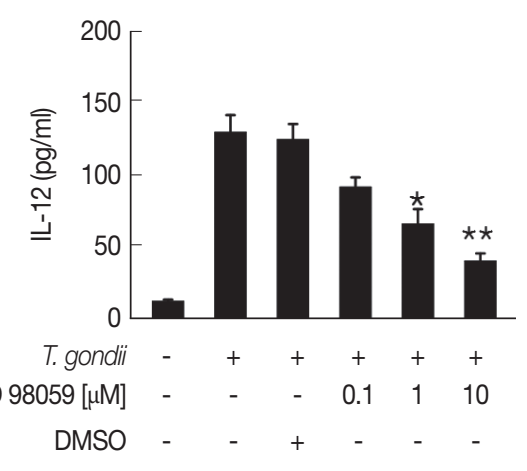

C

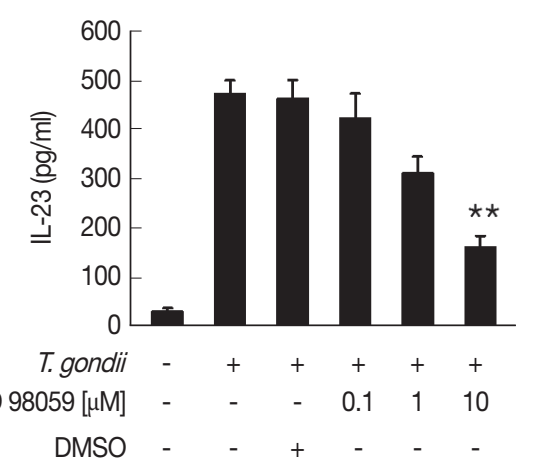

$E$

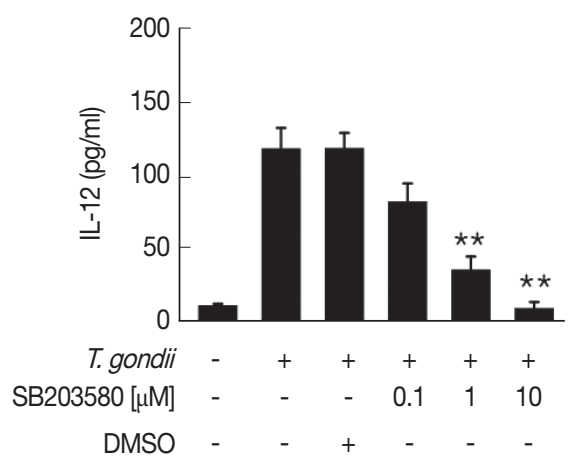

G

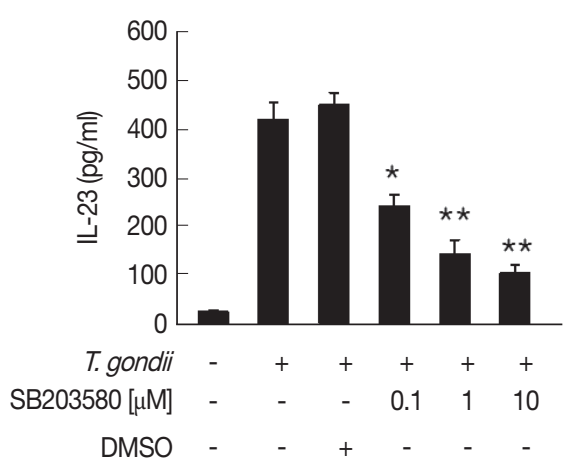

B

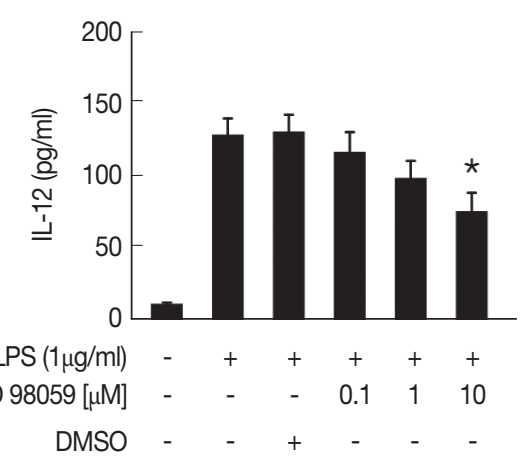

D

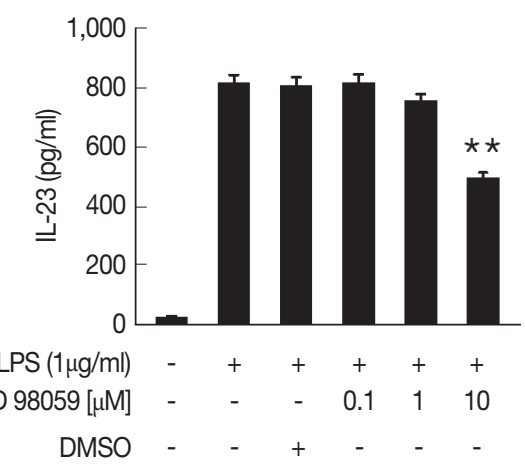

$\mathrm{F}$

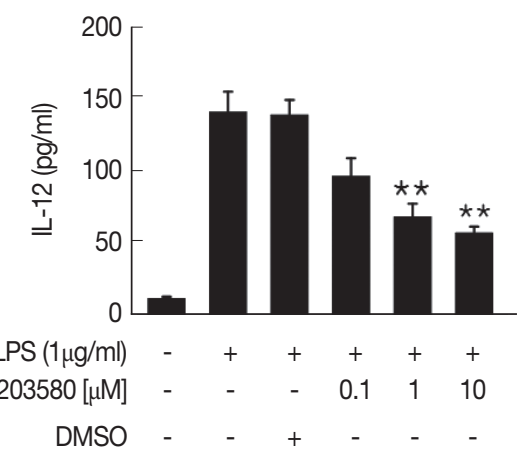

$\mathrm{H}$

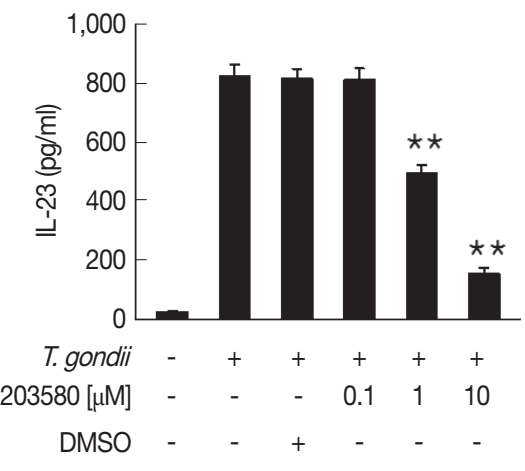

Fig. 5. Roles of the MAPK signaling pathways in T. gondii- and LPS-induced IL-12 and IL-23 production in human Jurkat T cells. Human Jurkat T cells were pretreated with various concentrations of the ERK1/2 inhibitor PD98059 (A-D), p38 MAPK inhibitor SB203580 (E-H). 

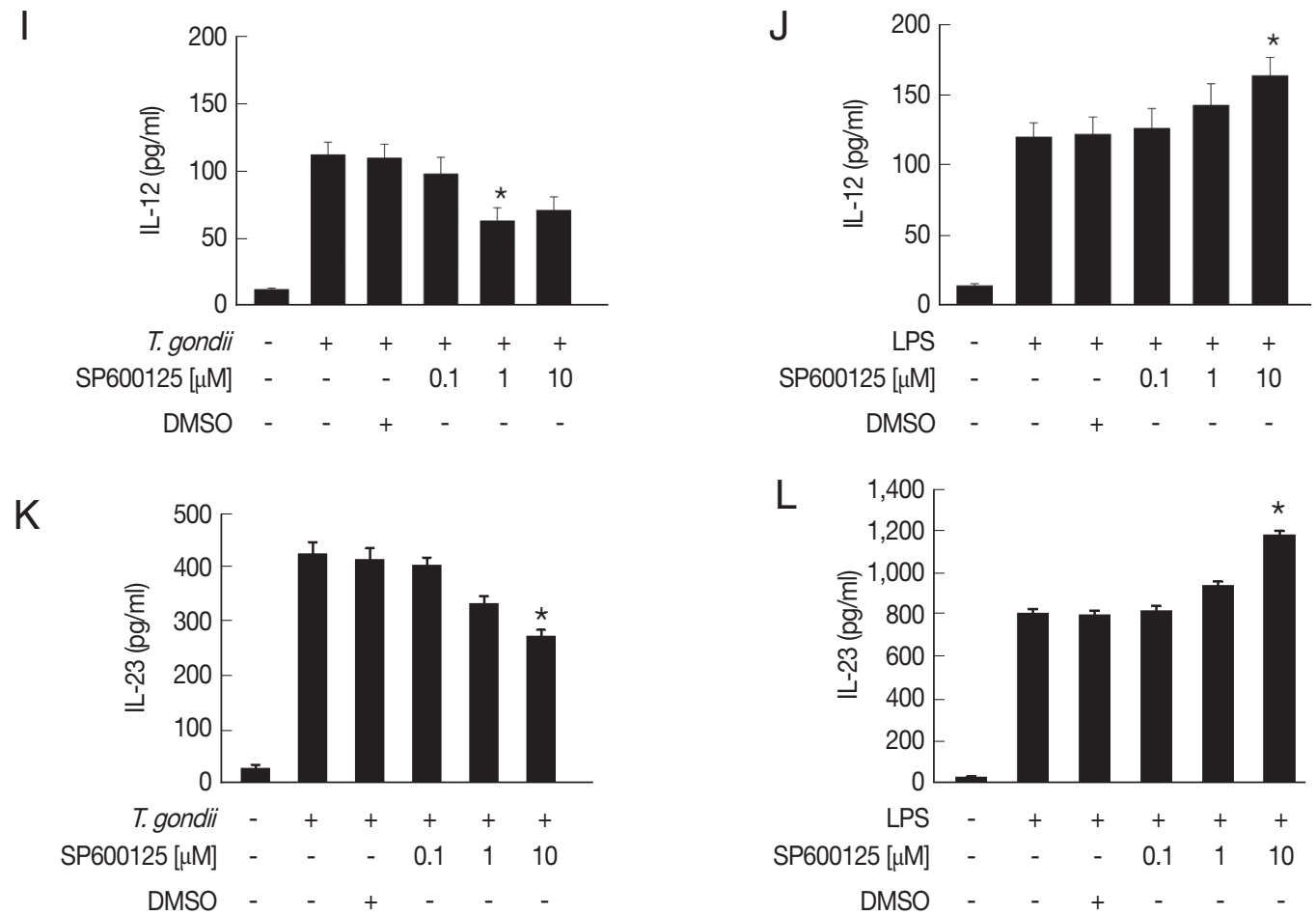

Fig. 5. (Continued) JNK1/2 inhibitor SP600125 (I-L) for $1 \mathrm{hr}$, and then infected with T. gondii RH or treated with LPS for 12 hr. IL-12 and IL-23 levels in the culture supernatants were analyzed by ELISA. One representative result of the 2 independents replicate is shown.

infected Jurkat T cells was inhibited by the PI3K inhibitors in a dose dependent manner, but increased in LPS-stimulated cells in a dose-dependent manner. Treatment with ERK1/2 and p38 MAPK inhibitors decreased IL-12 and IL-23 production in T. gondii- and LPS-stimulated cells in a dose-dependent manner; however, a JNK1/2 inhibitor exerted a different effect on IL-12 and IL-23 production from that of T. gondii and LPS. These data suggest that both T. gondii and LPS significantly upregulate IL12 and IL-23 production in Jurkat T cells; however, the roles of the PI3K and MAPK pathways in production of these cytokines differed according to the stimulus applied.

IL-12 and IL-23 are master regulators of innate and adaptive immunity [16], and they are primarily produced in antigenpresenting cells, such as macrophages and dendritic cells, after microbial challenge [10-12,17-19]. IL-12 induces protective responses to $\mathrm{T}$. gondii, including proliferation of $\mathrm{CD}^{+}$cytotoxic T-cells and IFN- $\gamma$ production by Th1 and NK cells [17]. IL-23 may play a limited protective role against intracellular pathogens, whereas IL-12 is indispensable for defense against toxoplasmosis [10]. We reported previously that IL-23 and IL-12 levels in THP-1 cells are significantly increased after stimulation with $T$. gondii antigens, including live tachyzoites, excretory/secretory products, or soluble antigen [11]. In the present study, we used Jurkat T cells, immortalized human leukemic T lymphocytes [20], to evaluate IL-12 and IL-23 production after stimulation with $T$. gondii and LPS. Our data showed that IL12 and IL-23 production in human Jurkat T cells was significantly induced from 30 min after stimulation with live T. gondii or LPS in a time- and dose-dependent manner. These data presented that functionally competent TLRs are also present on T lymphocytes such as innate immune cells (macrophages, neutrophils, dendritic cells and epithelial cells lining mucosal surfaces) and their activation either alone or in conjunction with concurrent TCR ligation brings about secretion of cytokines and chemokines from T cells [21-24]. Sharma et al. [24] reported the mechanism of action of Jurkat T-cell activation. The Src-kinases Lck and Fyn, which play a vital role in signal transduction through the TCR, are required for induction of the TLR-activated innate immune response in human $\mathrm{T}$ cells [24]. The results of these studies confirm that stimulation of $\mathrm{T}$ cells with T. gondii and LPS induces IL-12 and IL-23 production.

Next, we compared the phosphorylation patterns of components of the PI3K/AKT and MAPK pathways in various cell types after stimulation with T. gondii or LPS. Jurkat cells are also useful for evaluating stress-mediated pathways, including 
the p38 MAPK, JNK, and NF- $\mathrm{KB}$ signaling pathways [20]. Our data indicate that $T$. gondii and LPS induced the phosphorylation of AKT, ERK1/2, p38 MAPK, and JNK1/2 in human Jurkat T cells, similar to phosphorylation of AKT and MAPK subtypes in mouse bone marrow-derived macrophages after $T$. gondii infection and LPS stimulation $[5,14]$. These results suggest that the PI3K/AKT and MAPK pathways are rapidly activated in various cell types after treatment with T. gondii or LPS. We also evaluated the roles of the PI3K/AKT and MAPK pathways in the production of IL-12 and IL-23 by T cells stimulated with $T$. gondii or LPS. Our results indicate that IL-12 and IL-23 production in Jurkat T cells is positively regulated by ERK1/2 and p38 MAPK in response to both T. gondii and LPS. However, IL-12 and IL-23 production was positively regulated by the PI3K and JNK1/2 pathways in T. gondii-infected T cells but negatively regulated in LPS-stimulated cells. These results are in agreement with previous reports that IL-12 and IL-23 production is significantly increased in LPS-stimulated DCs after treatment with the PI3K inhibitor wortmannin compared to cells stimulated only with LPS [25], and IL-12 production in macrophages in response to T. gondii is dependent on the phosphorylation of p38 MAPK through TRAF6 [26]. However, Quan et al. [12] reported that IL-23 production is positively regulated by PI3K and ERK1/2 in THP-1 cells infected with T. gondii, whereas IL-12 production is positively regulated by $\mathrm{p} 38 \mathrm{MAPK}$ and JNK. These results indicate that the regulatory mechanisms of IL-12 and IL-23 production by the PI3K and MAPK pathways differ according to cell type and the applied stimulus.

In the present study, we investigated the roles of the PI3K/ AKT and MAPK pathways in the production of IL-12 and IL-23 by Jurkat $T$ cells stimulated with T. gondii or LPS. IL-12 and IL23 production was significantly upregulated in Jurkat $\mathrm{T}$ cells after stimulation with T. gondii or LPS. The PI3K and MAPK pathways were involved in regulation of IL 12 and IL-23 production in human Jurkat T cells, and T. gondii and LPS exerted different effects on the production of these cytokines. Our findings provide new insight into the regulation of $T$. gondiiand LPS-induced IL-23 and IL-12 production in Jurkat T cells.

\section{ACKNOWLEDGMENTS}

This work was supported by research fund of Chungnam National University.

\section{CONFLICT OF INTEREST}

We have no conflict of interest related to this study.

\section{REFERENCES}

1. Montoya JG, Liesenfeld O. Toxoplasmosis. Lancet 2004; 363: 1965-1976.

2. Denkers EY, Gazzinelli RT. Regulation and function of T-cellmediated immunity during Toxoplasma gondii infection. Clin Microbiol Rev 1998; 11: 569-588.

3. Jordan KA, Hunter CA. Regulation of CD8+ T cell responses to infection with parasitic protozoa. Exp Parasitol 2010; 126: 318325.

4. Rossol M, Heine H, Meusch U, Quandt D, Klein C, Sweet MJ, Hauschildt S. LPS-induced cytokine production in human monocytes and macrophages. Crit Rev Immunol 2011; 31: 379446.

5. Kim L, Butcher BA, Denkers EY. Toxoplasma gondii interferes with lipopolysaccharide-induced mitogen-activated protein kinase activation by mechanisms distinct from endotoxin tolerance. J Immunol 2004; 172: 3003-3010.

6. Vignali DA, Kuchroo VK. IL-12 family cytokines: immunological playmakers. Nat Immunol 2012; 13: 722-728.

7. Roses RE, Xu S, Xu M, Koldovsky U, Koski G, Czerniecki BJ. Differential production of IL-23 and IL-12 by myeloid-derived dendritic cells in response to TLR agonists. J Immunol 2008; 181 5120-5127.

8. Duvallet E, Semerano L, Assier E, Falgarone G, Boissier MC. Interleukin-23: a key cytokine in inflammatory diseases. Ann Med 2011; 43: 503-511.

9. Teng MW, Bowman EP, McElwee JJ, Smyth MJ, Casanova JL, Cooper AM, Cua DJ. IL-12 and IL-23 cytokines: from discovery to targeted therapies for immune-mediated inflammatory diseases. Nat Med 2015; 21: 719-729.

10. Lieberman LA, Cardillo F, Owyang AM, Rennick DM, Cua DJ, Kastelein RA, Hunter CA. IL-23 provides a limited mechanism of resistance to acute toxoplasmosis in the absence of IL-12. J Immunol 2004; 173: 1887-1893.

11. Quan JH, Zhou W, Cha GH, Choi IW, Shin DW, Lee YH. Kinetics of IL-23 and IL-12 secretion in response to Toxoplasma gondii antigens from THP-1 monocytic cells. Korean J Parasitol 2013; 51: 85-92.

12. Quan JH, Chu JQ, Kwon J, Choi IW, Ismail HA, Zhou W, Cha GH, Zhou Y, Yuk JM, Jo EK, Lee YH. Intracellular networks of the PI3K/AKT and MAPK pathways for regulating Toxoplasma gondii-induced IL-23 and IL-12 production in human THP-1 cells. PLoS One 2015; 10: e0141550.

13. Cantley LC. The phosphoinositide 3-kinase pathway. Science 2002; 296: 1655-1657.

14. Kim L, Denkers EY. Toxoplasma gondii triggers Gi-dependent PI 3-kinase signaling required for inhibition of host cell apoptosis. 
J Cell Sci 2006; 119: 2119-2126.

15. Denkers EY, Butcher BA, Del Rio L, Kim L. Manipulation of mitogen-activated protein kinase/nuclear factor- $\kappa \mathrm{B}$-signaling cascades during intracellular Toxoplasma gondii infection. Immunol Rev 2004; 201: 191-205.

16. Langrish CL, McKenzie BS, Wilson NJ, de Waal Malefyt R, Kastelein RA, Cua DJ. IL-12 and IL-23: master regulators of innate and adaptive immunity. Immunol Rev 2004; 202: 96-105.

17. Sher A, Collazzo C, Scanga C, Jankovic D, Yap G, Aliberti J. Induction and regulation of IL-12-dependent host resistance to Toxoplasma gondii. Immunol Res 2003; 27: 521-528.

18. Muñoz M, Heimesaat MM, Danker K, Struck D, Lohmann U, Plickert R, Bereswill S, Fischer A, Dunay IR, Wolk K, Loddenkemper C, Krell HW, Libert C, Lund LR, Frey O, Hölscher C, Iwakura Y, Ghilardi N, Ouyang W, Kamradt T, Sabat R, Liesenfeld O Interleukin (IL)-23 mediates Toxoplasma gondii-induced immunopathology in the gut via matrixmetalloproteinase- 2 and IL-22 but independent of IL-17. J Exp Med 2009; 206: 30473059.

19. Yarovinsky F. Innate immunity to Toxoplasma gondii infection. Nat Rev Immunol 2014; 14: 109-121.

20. Abraham RT, Weiss A. Jurkat T cells and development of the T- cell receptor signalling paradigm. Nat Rev Immunol 2004; 4: 301-308

21. Kabelitz D. Expression and function of Toll-like receptors in T lymphocytes. Curr Opin Immunol 2007; 19: 39-45.

22. Reynolds JM, Dong C. Toll-like receptor regulation of effector $\mathrm{T}$ lymphocyte function. Trends Immunol 2013; 34: 511-519.

23. Akhade AS, Qadri A. T-cell receptor activation of human $\mathrm{CD}_{4}^{+} \mathrm{T}$ cells shifts the innate TLR response from CXCL8 ${ }^{\text {hi }}$ IFN- $\gamma^{\text {null }}$ to CXCL $8^{\text {lo }}$ IFN- $\gamma^{\text {hi }}$. Eur J Immunol 2015; 45: 2628-2637.

24. Sharma N, Akhade AS, Qadri A. Src kinases central to T-cell receptor signaling regulate TLR-activated innate immune responses from human T cells. Innate Immun 2016; 22: 238-244.

25. Schaper K, Kietzmann M, Bäumer W. Sphingosine-1-phosphate differently regulates the cytokine production of IL-12, IL-23 and IL-27 in activated murine bone marrow derived dendritic cells. Mol Immunol 2014; 59: 10-18.

26. Mason NJ, Fiore J, Kobayashi T, Masek KS, Choi Y, Hunter CA. TRAF6-dependent mitogen-activated protein kinase activation differentially regulates the production of interleukin- 12 by macrophages in response to Toxoplasma gondii. Infect Immunol 2004; 72: 5662-5667. 